\title{
ESTIMATES OF GREEN FUNCTIONS AND HARMONIC MEASURES FOR ELLIPTIC OPERATORS WITH SINGULAR DRIFT TERMS
}

\author{
Abdoul Ifra ANd Lotfi Riahi
}

\begin{abstract}
In this paper, we prove the existence and uniqueness of the continuous Green function $G$ for the elliptic operator $L=\operatorname{div}\left(A(x) \nabla_{x}\right)+$ $B(x) \cdot \nabla_{x}$ with singular drift term $B$ on a $C^{1,1}$ bounded domain $D$ in $\mathbb{R}^{n}, n \geq 3$, and its comparability to the Green function $G_{0}$ of $L_{0}=\operatorname{div}\left(A(x) \nabla_{x}\right)$. Basing on this result we establish the equivalence of the $L$-harmonic measure and the surface measure on $\partial D$. These results extend some first ones proved for elliptic operators with less singular drift terms.
\end{abstract}

\section{Introduction}

In this paper we will interest with the following elliptic operator

$$
L=\operatorname{div}\left(A(x) \nabla_{x}\right)+B(x) \cdot \nabla_{x}
$$

on a $C^{1,1}$ bounded domain $D$ in $\mathbb{R}^{n}, n \geq 3$. Our aim is to investigate the existence and uniqueness of the continuous $L$-Green function $G$ and its comparability to the Green function $G_{0}$ of $L_{0}=\operatorname{div}\left(A(x) \nabla_{x}\right)$, where $A$ is a uniformly elliptic matrix with Lipschitz-continuous coefficients and the drift term $B$ is in a class $\mathcal{K}_{\text {loc }}(D)$ generalizing the well known Kato class $K_{n+1}$ used in [9]. Basing on the comparability of the Green functions we will be able to estimate the $L$-Poisson kernel which allow us to prove that the $L$-harmonic measure and the surface measure are mutually absolutely continuous on $\partial D$. Consequently, the operators $L$ and $L_{0}$ have the same potential theory on $D$. Before describing the body of this paper, we first recall that the same results are studied in $[\mathbf{4}]$ and $[\mathbf{1 5}]$ for more general elliptic operators but with coefficients Hölder continuous

2000 Mathematics Subject Classification. 35J15, 31B25, 31B05, 35-99.

Key words. Elliptic operator, drift term, Green function, Poisson kernel, harmonic measure, Kato class. 
up to the boundary of $D$. Subsequently, Cranston and Zhao [9] studied the operator $L=-\Delta_{x}+B(x) \cdot \nabla_{x}$ and proved, by a probabilistic approach, the same results when $|B|^{2} \in K_{n}$ and $|B| \in K_{n+1}$ (see Theorem 3.13 in $[\mathbf{9}])$. In particular our results extend this theorem to a large class of elliptic operators with more singular drift terms. Recently, Ancona [5] studied the equivalence of Green functions for more general elliptic operators on manifolds or domains. However, in the Euclidian case (see Theorems 9.1 and 9.1' in [5]), his results do not cover the ones proved here. In fact, we will provide (see Proposition 4.4) examples of vector fields in the class $\mathcal{K}_{\text {loc }}(D)$ which do not satisfy the conditions required by Ancona [5]. For other results concerning stability of Green function under perturbations we refer the reader to $[\mathbf{1}],[\mathbf{6}],[\mathbf{8}],[\mathbf{1 2}]$, $[\mathbf{1 3}],[\mathbf{1 6}]-[\mathbf{2 5}],[\mathbf{2 7}]$ and $[\mathbf{2 8}]$.

Our paper is organized as follows. In Section 2, we give some notations and we state some known results. In Section 3, we prove a $3 G_{0}$-type inequality which is the key in proving our main result in the next. In Section 4 , we introduce the class $\mathcal{K}_{\text {loc }}(D)$ and we study some of its properties. In particular we prove that $\mathcal{K}_{\text {loc }}(D)$ is more general than the Kato class $K_{n+1}$. In Section 5, we prove the existence and uniqueness of the continuous $L$-Green function $G$ when the vector $B$ is in the class $\mathcal{K}_{\text {loc }}(D)$. In Section 6 , we apply the previous results to prove the equivalence of the $L$-harmonic measure and the surface measure on $\partial D$.

Throughout the paper the letter $C$ denotes a generic positive constant which may vary in value from line to line.

\section{Notations and known results}

Throughout this paper $D$ denotes a $C^{1,1}$ bounded domain in $\mathbb{R}^{n}$, $n \geq 3$. This means that for all $z \in \partial D$, there exists $r_{0}>0$ such that $B\left(z, r_{0}\right) \cap \partial D$ is the graph of a $C^{1,1}$-function. As already mentioned, we will consider the elliptic operator

$$
L=L_{0}+B(x) \cdot \nabla_{x},
$$

where $L_{0}=\operatorname{div}\left(A(x) \nabla_{x}\right)$, on $D$. The matrix $A(x)=\left(a_{i j}(x)\right)_{1 \leq i, j \leq n}$ is assumed to be uniformly elliptic i.e. $\mu^{-1}\|\xi\|^{2} \leq\langle A(x) \xi, \xi\rangle \leq \mu\|\xi\|^{2}$, for all $x \in D, \xi \in \mathbb{R}^{n}$ and some $\mu \geq 1$ with $\mu$-Lipschitz continuous coefficients and the vector $B=B(x) \in L_{\mathrm{loc}}^{1}(D)$. We will denote by $G_{0}$ the $L_{0}$-Green function on $D$. For $x \in D$, let $d(x)=d(x, \partial D)$ denotes the distance from $x$ to the boundary of $D$. We recall the following useful estimates proved in $[\mathbf{1 1}],[\mathbf{1 4}],[\mathbf{2 6}]$ and $[\mathbf{2 7}]$. 
Theorem 2.1. There exists a constant $C=C(n, \mu, D)$ such that, for all $x, y \in D$,

1)

$$
\frac{1}{C} N(x, y) \leq G_{0}(x, y) \leq C N(x, y)
$$

where

$$
N(x, y)=\min \left(1, \frac{d(x)}{|x-y|}\right) \min \left(1, \frac{d(y)}{|x-y|}\right) \frac{1}{|x-y|^{n-2}},
$$

$$
\left|\nabla_{x} G_{0}(x, y)\right| \leq C \min \left(1, \frac{d(y)}{|x-y|}\right) \frac{1}{|x-y|^{n-1}} .
$$

The dependence of the constant $C$ on $D$ is only on the diameter of $D$ and the curvature of $\partial D$.

\section{A $3 G_{0}$-type inequality}

In this section, we prove the following fundamental inequality which is the key in proving our main result in the next. This inequality is reminiscent of the $3 G_{0}$-inequality proved in [22] and [24] on $C^{1,1}$-domains and in [23] on Lipschitz domains which played an important role to study Schrödinger equations.

For $x, z \in D$, let

$$
\Gamma(x, z)=\min \left(1, \frac{d(z)}{|x-z|}\right) \frac{1}{|x-z|^{n-1}} .
$$

Theorem 3.1. There exists a constant $C_{0}=C_{0}(n, \mu, D)>0$ such that for all $x, y, z \in D$, we have

$$
\frac{G_{0}(x, z)\left|\nabla_{z} G_{0}(z, y)\right|}{G_{0}(x, y)} \leq C_{0}(\Gamma(x, z)+\Gamma(y, z)) .
$$

Proof: From Theorem 2.1, we have

$$
\frac{G_{0}(x, z)\left|\nabla_{z} G_{0}(z, y)\right|}{G_{0}(x, y)} \leq C \omega(x, z, y) \frac{1}{|z-y|}\left(\frac{|x-y|}{|x-z||z-y|}\right)^{n-2},
$$

where

$$
\omega(x, z, y)=\frac{\min \left(1, \frac{d(x)}{|x-z|}\right) \min \left(1, \frac{d(z)}{|x-z|}\right) \min \left(1, \frac{d(y)}{|z-y|}\right)}{\min \left(1, \frac{d(x)}{|x-y|}\right) \min \left(1, \frac{d(y)}{|x-y|}\right)} .
$$


Case 1: $|x-z| \geq|z-y|$.

We have $|x-y| \leq|x-z|+|z-y| \leq 2|x-z|$ and so

$$
\left(\frac{|x-y|}{|x-z||z-y|}\right)^{n-2} \leq \frac{2^{n-2}}{|z-y|^{n-2}} .
$$

Moreover, by using the inequalities $\frac{a b}{a+b} \leq \min (a, b) \leq 2 \frac{a b}{a+b}$, for all $a, b>0$ and $|d(z)-d(y)| \leq|z-y|$, we have

$$
\begin{aligned}
\omega(x, z, y) & \leq 2 \frac{\min \left(1, \frac{d(z)}{|x-z|}\right)}{\min \left(1, \frac{d(y)}{|x-y|}\right)} \min \left(1, \frac{d(y)}{|z-y|}\right) \\
& \leq 8 \frac{d(z)}{d(y)} \min \left(1, \frac{d(y)}{|z-y|}\right) \\
& \leq 16 \min \left(1, \frac{d(z)}{|z-y|}\right) .
\end{aligned}
$$

Combining (3.1)-(3.3), we obtain

$$
\frac{G_{0}(x, z)\left|\nabla_{z} G_{0}(z, y)\right|}{G_{0}(x, y)} \leq C \Gamma(y, z) .
$$

Case 2: $|z-y| \geq|x-z|$.

We have $|x-y| \leq|x-z|+|z-y| \leq 2|z-y|$ and so

$$
\left(\frac{|x-y|}{|x-z||z-y|}\right)^{n-2} \leq \frac{2^{n-2}}{|x-z|^{n-2}} .
$$

Moreover, we have

$$
\begin{aligned}
\omega(x, z, y) & \leq 2 \frac{\min \left(1, \frac{d(x)}{|x-z|}\right)}{\min \left(1, \frac{d(x)}{|x-y|}\right)} \min \left(1, \frac{d(z)}{|x-z|}\right) \\
& \leq 8 \frac{d(x)+|x-y|}{d(x)+|x-z|} \min \left(1, \frac{d(z)}{|x-z|}\right) \\
& \leq 8\left(1+2 \frac{|z-y|}{|x-z|}\right) \min \left(1, \frac{d(z)}{|x-z|}\right) \\
& \leq 24 \frac{|z-y|}{|x-z|} \min \left(1, \frac{d(z)}{|x-z|}\right) .
\end{aligned}
$$


Combining (3.1)-(3.5), we obtain

$$
\frac{G_{0}(x, z)\left|\nabla_{z} G_{0}(z, y)\right|}{G_{0}(x, y)} \leq C \Gamma(x, z) .
$$

\section{The class $\mathcal{K}_{\text {loc }}(D)$}

In order to introduce the class $\mathcal{K}_{\text {loc }}(D)$, we shall first recall the well known Kato classes $K_{n}$ and $K_{n}^{\text {loc }}($ see $[2])$.

Definition 4.1. Let $V$ be a Borel measurable function on $D$. We say that $V$ is in the local Kato class $K_{n}^{\text {loc }}$ if it satisfies

$$
\lim _{r \rightarrow 0} \sup _{x \in E} \int_{D \cap(|x-z|<r)} \frac{|V(z)|}{|x-z|^{n-2}} d z=0,
$$

for every compact subset $E \subset D$. We have $K_{n}^{\text {loc }} \subset L_{\text {loc }}^{1}(D)$.

We say that $V$ is in the Kato class $K_{n}$ if in the above definition $\sup _{x \in E}$ is replaced by $\sup _{x \in D}$. Clearly, if $V \in K_{n}$, then by a compactness argument (since $\bar{D}$ is bounded), we have

$$
\sup _{x \in \bar{D}} \int_{D} \frac{|V(z)|}{|x-z|^{n-2}} d z<+\infty .
$$

In particular, $K_{n} \subset L^{1}(D)$.

The Kato class $K_{n}^{\text {loc }}$ introduced in [2] by Aizenman and Simon is considered as the biggest possible space of potentials $V=V(x)$ so that the Harnack inequalities and the continuity of solutions for the Schrödinger operator $-\Delta+V(x)$ hold. It is also used later by several authors to study the potential theory of the Schrödinger operator (see [27], [8], [7], [28] and the references therein). For the operators with drift terms $L=-\Delta+B(x) \cdot \nabla_{x}$, Cranston and Zhao [9] proved that when $|B| \in K_{n+1}$ and $|B|^{2} \in K_{n}$, the Green functions and harmonic measures for $L$ and $\Delta$ are equivalent.

Definition 4.2. Let $B$ be a Borel measurable vector field on $D$. We say that $B$ is in the class $\mathcal{K}_{\text {loc }}(D)$ if $B \in K_{n+1}^{\text {loc }}$ and satisfies

$$
\|B\| \equiv \sup _{x \in D} \int_{D} \Gamma(x, z)|B(z)| d z<+\infty .
$$

Obviously, $K_{n+1} \subset \mathcal{K}_{\text {loc }}(D) \subset K_{n+1}^{\text {loc }}$. In what follows we study some properties of the class $\mathcal{K}_{\text {loc }}(D)$. In particular we prove that $\mathcal{K}_{\text {loc }}(D)$ contains more singular vector fields than the Kato class $K_{n+1}$ used by Cranston and Zhao in [9]. 
Proposition 4.3. Let $D=B(0,1)$ be the unit ball. Then, for all $1<$ $\lambda<2$, all $\vec{u} \in \mathbb{R}^{n}, \vec{u} \neq 0$, the radial vector field on $D$,

$$
B_{\lambda}(r)=\frac{1}{(1-r)\left(1+\log \frac{1}{1-r}\right)^{\lambda}} \vec{u} \in \mathcal{K}_{\mathrm{loc}}(D) \backslash K_{n+1} .
$$

Proof: Consider a spherical coordinate system $\left(r, \theta_{1}, \ldots, \theta_{n-1}\right)$ such that $x=(|x|, 0, \ldots, 0)$ and set $|x|=r_{0}$, we have

$$
\begin{aligned}
& \sup _{x \in \bar{D}} \int_{D} \frac{\left|B_{\lambda}(z)\right|}{|x-z|^{n-1}} d z \\
& \quad=C \sup _{0 \leq r_{0} \leq 1} \int_{0}^{1} r^{n-1}\left|B_{\lambda}(r)\right| \int_{0}^{\pi} \frac{\left(\sin \theta_{1}\right)^{n-2}}{\left(r^{2}+r_{0}^{2}-2 r r_{0} \cos \theta_{1}\right)^{\frac{n-1}{2}}} d \theta_{1} d r .
\end{aligned}
$$

By making the change of variables $t=\tan \frac{\theta_{1}}{2}$ and letting $\alpha=\frac{r+r_{0}}{\left|r-r_{0}\right|}$, we obtain

$$
\sup _{x \in \bar{D}} \int_{D} \frac{\left|B_{\lambda}(z)\right|}{|x-z|^{n-1}} d z=C \sup _{0 \leq r_{0} \leq 1} \int_{0}^{1} \frac{r^{n-1}\left|B_{\lambda}(r)\right|}{\left|r-r_{0}\right|^{n-1}} \varphi(\alpha) d r
$$

where

$$
\varphi(\alpha)=\int_{0}^{\infty} \frac{t^{n-2}}{\left(1+t^{2}\right)^{\frac{n-1}{2}}\left(1+\alpha^{2} t^{2}\right)^{\frac{n-1}{2}}} d t .
$$

Moreover, we have

$$
\begin{aligned}
\varphi(\alpha) & =\int_{0}^{\infty} \frac{t^{n-2}}{\left(1+t^{2}\right)^{\frac{n-1}{2}}\left(1+\alpha^{2} t^{2}\right)^{\frac{n-1}{2}}} d t \\
& \geq \frac{C}{\alpha^{n-1}} \int_{1 / \alpha}^{1} \frac{1}{t} d t=\frac{C}{\alpha^{n-1}} \log \alpha .
\end{aligned}
$$

Combining (4.1) and (4.2), we obtain

$$
\begin{aligned}
\sup _{x \in \bar{D}} \int_{D} \frac{\left|B_{\lambda}(z)\right|}{|x-z|^{n-1}} d z & \geq C \sup _{0 \leq r_{0} \leq 1} \int_{0}^{1} \frac{r^{n-1}\left|B_{\lambda}(r)\right|}{\left(r+r_{0}\right)^{n-1}} \log \frac{r+r_{0}}{\left|r-r_{0}\right|} d r \\
& \geq C \int_{0}^{1} r^{n-1}\left|B_{\lambda}(r)\right| \log \frac{1}{1-r} d r=+\infty .
\end{aligned}
$$

Now, we prove that $B_{\lambda}$ is in the class $\mathcal{K}_{\text {loc }}(D)$. Clearly $B_{\lambda}$ is locally bounded and so $B_{\lambda} \in K_{n+1}^{\text {loc }}$. We will show that $\left\|B_{\lambda}\right\|<+\infty$. By using 
the same spherical coordinate system as above, we obtain

$$
\begin{aligned}
\left\|B_{\lambda}\right\| & \equiv \sup _{x \in D} \int_{D} \Gamma(x, z)\left|B_{\lambda}(z)\right| d z \\
& =C \sup _{0 \leq r_{0}<1} \int_{0}^{1} \frac{r^{n-1}\left|B_{\lambda}(r)\right|}{\left|r-r_{0}\right|^{n-1}} \psi(\alpha) d r,
\end{aligned}
$$

where

$\psi(\alpha)=\int_{0}^{\infty} \min \left(1, \frac{(1-r)\left(1+t^{2}\right)^{1 / 2}}{\left|r-r_{0}\right|\left(1+\alpha^{2} t^{2}\right)^{1 / 2}}\right) \frac{t^{n-2}}{\left(1+t^{2}\right)^{\frac{n-1}{2}}\left(1+\alpha^{2} t^{2}\right)^{\frac{n-1}{2}}} d t$.

By recalling that, for $a>0,(1 \vee a)^{2} \leq 1+a^{2} \leq 2(1 \vee a)^{2}$, it follows that

$$
\begin{aligned}
& \psi(\alpha)= \int_{0}^{\infty} \min \left(1, \frac{(1-r)\left(1+t^{2}\right)^{1 / 2}}{\left|r-r_{0}\right|\left(1+\alpha^{2} t^{2}\right)^{1 / 2}}\right) \frac{t^{n-2}}{\left(1+t^{2}\right)^{\frac{n-1}{2}}\left(1+\alpha^{2} t^{2}\right)^{\frac{n-1}{2}}} d t \\
& \leq C\left[\min \left(1, \frac{1-r}{\left|r-r_{0}\right|}\right) \int_{0}^{1 / \alpha} t^{n-2} d t\right. \\
&+\frac{1}{\alpha^{n-1}} \int_{1 / \alpha}^{1} \min \left(1, \frac{1-r}{\left(r+r_{0}\right) t}\right) \frac{1}{t} d t \\
&\left.+\min \left(1, \frac{1-r}{r+r_{0}}\right) \frac{1}{\alpha^{n-1}} \int_{1}^{\infty} \frac{1}{t^{n}} d t\right] \\
& \leq \frac{C}{\alpha^{n-1}}\left[\min \left(1, \frac{1-r}{\left|r-r_{0}\right|}\right)+\int_{1 / \alpha}^{1} \min \left(1, \frac{1-r}{\left(r+r_{0}\right) t}\right) \frac{1}{t} d t\right] .
\end{aligned}
$$

On the other hand

$$
\begin{aligned}
\int_{1 / \alpha}^{1} \min \left(1, \frac{1-r}{\left(r+r_{0}\right) t}\right) \frac{1}{t} d t & \leq 2 \int_{1 / \alpha}^{1} \frac{1-r}{\left(1-r+\left(r+r_{0}\right) t\right) t} d t \\
& =2 \int_{\frac{\left|r-r_{0}\right|}{1-r}}^{\frac{r+r_{0}}{1-r}} \frac{1}{(s+1) s} d s \\
& =2 \log \left[\left(\frac{r+r_{0}}{1+r_{0}}\right)\left(1+\frac{1-r}{\left|r-r_{0}\right|}\right)\right] \\
& \leq 2 \log \left(1+\frac{1-r}{\left|r-r_{0}\right|}\right) .
\end{aligned}
$$


Combining (4.4) and (4.5) and recalling that $\min (1, t) \leq \log (1+t)$, for all $t \geq 0$, it follows that

$$
\psi(\alpha) \leq \frac{C}{\alpha^{n-1}} \log \left(1+\frac{1-r}{\left|r-r_{0}\right|}\right) .
$$

Combining (4.3) and (4.6), we obtain

$$
\begin{aligned}
\left\|B_{\lambda}\right\| & \leq C \sup _{0 \leq r_{0} \leq 1} \int_{0}^{1} \frac{r^{n-1}\left|B_{\lambda}(r)\right|}{\left(r+r_{0}\right)^{n-1}} \log \left(1+\frac{1-r}{\left|r-r_{0}\right|}\right) d r \\
& \leq C \sup _{0 \leq r_{0} \leq 1} \int_{0}^{1}\left|B_{\lambda}(r)\right| \log \left(1+\frac{1-r}{\left|r-r_{0}\right|}\right) d r .
\end{aligned}
$$

For $r_{0} \in[0,1]$, set

$$
\begin{aligned}
F\left(r_{0}\right) & =\int_{0}^{1}\left|B_{\lambda}(r)\right| \log \left(1+\frac{1-r}{\left|r-r_{0}\right|}\right) d r \\
& =\int_{0}^{1} \frac{\log \left(1+\frac{1-r}{\left|r-r_{0}\right|}\right)}{(1-r)\left(1+\log \frac{1}{1-r}\right)^{\lambda}} d r
\end{aligned}
$$

By making the change of variables $s=\frac{1-r_{0}}{1-r}$, we have

$$
F\left(r_{0}\right)=\int_{1-r_{0}}^{\infty} \frac{\log \left(1+\frac{1}{|s-1|}\right)}{s\left(1+\log \frac{s}{1-r_{0}}\right)^{\lambda}} d s
$$

For $r_{0} \in\left[0, \frac{1}{2}\right]$, we have

$$
F\left(r_{0}\right) \leq \int_{1 / 2}^{\infty} \frac{\log \left(1+\frac{1}{|s-1|}\right)}{s} d s=C<+\infty .
$$

For $r_{0} \in\left[\frac{1}{2}, 1\right]$, we have

$$
\begin{aligned}
F\left(r_{0}\right) & =\int_{1-r_{0}}^{\infty} \frac{\log \left(1+\frac{1}{|s-1|}\right)}{s(1+\log s)^{\lambda}} d s \\
& =\int_{1-r_{0}}^{1 / 2} \cdots d s+\int_{1 / 2}^{\infty} \cdots d s \equiv I_{1}+I_{2} .
\end{aligned}
$$


We have

$$
\begin{aligned}
I_{1} & =\int_{1-r_{0}}^{1 / 2} \frac{\log \left(1+\frac{1}{|s-1|}\right)}{s\left(1+\log \frac{s}{1-r_{0}}\right)^{\lambda}} d s \\
& \leq \log 3 \int_{1-r_{0}}^{1 / 2} \frac{1}{s\left(1+\log \frac{s}{1-r_{0}}\right)^{\lambda}} d s \\
& \leq \log 3 \int_{1}^{\infty} \frac{1}{t(1+\log t)^{\lambda}} d t=C<+\infty,
\end{aligned}
$$

and

$$
\begin{aligned}
I_{2} & =\int_{1 / 2}^{\infty} \frac{\log \left(1+\frac{1}{|s-1|}\right)}{s\left(1+\log \frac{s}{1-r_{0}}\right)^{\lambda}} d s \\
& \leq \int_{1 / 2}^{\infty} \frac{\log \left(1+\frac{1}{|s-1|}\right)}{s} d s=C<+\infty .
\end{aligned}
$$

Combining (4.7)-(4.11), we get

$$
\left\|B_{\lambda}\right\| \leq C<+\infty
$$

In what follows we will provide examples of vector fields in the class $K_{n+1}$ which do not satisfy the conditions required by Ancona in [5]. To this end we first recall (see Theorem 9.1' in [5]) that for our operator the results of Ancona are applied to vectors $B$ in $L_{\mathrm{loc}}^{p}(D)$, for $p>n$ satisfying:

$$
(d(x))^{1-\frac{n}{p}}\|B\|_{L^{p}\left(B\left(x, \frac{d(x)}{2}\right)\right)} \leq \varphi(d(x)),
$$

for all $x \in D$ with $\varphi$ is a nondecreasing function on $(0,+\infty)$ and satisfies $\int_{0}^{1} \frac{\varphi(s)}{s} d s<\infty$.

We have the following:

Proposition 4.4. Let $D=B(0,1)$ be the unit ball in $\mathbb{R}^{n}$. For all $\lambda>1 / n$, the radial vector field $B_{\lambda}$ defined on $D$ by

$$
B_{\lambda}(r)= \begin{cases}\left(\frac{1}{2}-r\right)^{-\frac{1}{n}}\left|\log \left(\frac{1}{2}-r\right)\right|^{-\lambda} & \text { if } 0 \leq r<\frac{1}{2} \\ 0 & \text { if } \frac{1}{2} \leq r<1\end{cases}
$$

is in $K_{n+1}$ and does not belong to any $L_{\mathrm{loc}}^{p}(D)$ with $p>n$. 
Proof: Clearly, for $p>n$,

$$
\int_{0}^{\frac{1}{2}}\left|B_{\lambda}(r)\right|^{p} d r=\int_{0}^{\frac{1}{2}} \frac{d r}{\left(\frac{1}{2}-r\right)^{\frac{p}{n}}\left|\log \left(\frac{1}{2}-r\right)\right|^{p \lambda}}=+\infty
$$

and so $B_{\lambda}$ does not belong to $L_{\text {loc }}^{p}(D)$.

On the other hand, we have

$$
\int_{0}^{1}\left|B_{\lambda}(r)\right|^{n} d r=\int_{0}^{\frac{1}{2}} \frac{d r}{\left(\frac{1}{2}-r\right)\left|\log \left(\frac{1}{2}-r\right)\right|^{n \lambda}}<+\infty
$$

and so $B_{\lambda} \in L^{n}(D)$.

Hence, for a given $\varepsilon>0$, there is $\alpha=\alpha(\varepsilon)>0$ such that

$$
\left(\int_{\frac{1}{2}-\alpha}^{\frac{1}{2}}\left|B_{\lambda}(r)\right|^{n} d r\right)^{\frac{1}{n}}<\varepsilon
$$

Thus,

$$
\begin{aligned}
\int_{D \cap(|x-z| \leq r)} \frac{\left|B_{\lambda}(z)\right|}{|x-z|^{n-1}} d z= & \int_{D \cap(|x-z| \leq r) \cap\left(\frac{1}{2}-\alpha \leq|z|<\frac{1}{2}\right)} \frac{\left|B_{\lambda}(z)\right|}{|x-z|^{n-1}} d z \\
& +\int_{D \cap(|x-z| \leq r) \cap\left(|z| \leq \frac{1}{2}-\alpha\right)} \frac{\left|B_{\lambda}(z)\right|}{|x-z|^{n-1}} d z \\
\equiv & I_{1}(x)+I_{2}(x) .
\end{aligned}
$$

By the Hölder inequality, we have

$$
I_{1}(x) \leq \omega_{n}^{1-\frac{1}{n}}\left(\int_{\frac{1}{2}-\alpha}^{\frac{1}{2}}\left|B_{\lambda}(r)\right|^{n} d r\right)^{\frac{1}{n}} \leq \omega_{n}^{1-\frac{1}{n}} \varepsilon,
$$

where $\omega_{n}$ is the volume of the unit sphere in $\mathbb{R}^{n}$.

Moreover

$$
I_{2}(x) \leq \frac{\alpha^{-\frac{1}{n}}}{(\log 2)^{\lambda}} \int_{|x-z|<r} \frac{d z}{|x-z|^{n-1}}=\frac{\alpha^{-\frac{1}{n}}}{(\log 2)^{\lambda}} \omega_{n} r .
$$

Combining (4.12)-(4.14), we obtain, for $r$ sufficiently small,

$$
\sup _{x \in D} \int_{D \cap(|x-z| \leq r)} \frac{\left|B_{\lambda}(z)\right|}{|x-z|^{n-1}} d z \leq 2 \omega_{n}^{1-\frac{1}{n}} \varepsilon,
$$

and so $B_{\lambda} \in K_{n+1}$. 


\section{The $L$-Green function $G$}

In this section we prove the existence of the $L$-Green function $G$ on $D$ and its comparability to $G_{0}$, when the drift term $B$ is in the class $\mathcal{K}_{\text {loc }}(D)$. Following the classical potential theory a Borel measurable function $G: D \times D \rightarrow] 0,+\infty]$ is called a Green function for the operator $L$ on $D$ if it satisfies for all $y \in D, L G(\cdot, y)=-\varepsilon_{y}$ in the distributional sense, where $\varepsilon_{y}$ is the Dirac measure at $y$ and $G(\cdot, y)$ vanishes on $\partial D$. We say that $G$ is continuous if it is continuous outside the diagonal. Our main result is the following:

Theorem 5.1. Let $B \in \mathcal{K}_{\text {loc }}(D)$ with $\|B\|<1 / 4 C_{0}$. Then, there exists a unique continuous $L$-Green function $G$ on $D$ comparable to $G_{0}$, i.e. there is a constant $C=C(n, \mu, D,\|B\|)>0$ such that

$$
\frac{1}{C} G_{0} \leq G \leq C G_{0}
$$

Proof: Let $B$ as in the theorem. From Theorem 3.1, we have, for $x, y \in D$,

$$
\begin{aligned}
& \int_{D} G_{0}(x, z) \mid B(z)|| \nabla_{z} G_{0} \mid(z, y) d z \\
& \leq C_{0} \int_{D}(\Gamma(x, z)+\Gamma(y, z))|B(z)| d z G_{0}(x, y) \\
& \leq 2 C_{0}\|B\| G_{0}(x, y) .
\end{aligned}
$$

Since $G_{0}<\infty$ outside the diagonal then for all $x \neq y \in D$, let

$$
G_{0} *\left(B \nabla G_{0}\right)(x, y)=\int_{D} G_{0}(x, z) B(z) \nabla_{z} G_{0}(z, y) d z .
$$

In view of (5.1), we may define, for any $m \in \mathbb{N}, G_{0} *\left(B \nabla G_{0}\right)^{* m}$ outside the diagonal by $G_{0} *\left(B \nabla G_{0}\right)^{* m}=\left(G_{0} *\left(B \nabla G_{0}\right)^{* m-1}\right) *\left(B \nabla G_{0}\right)$ with $G_{0} *\left(B \nabla G_{0}\right)^{* 0}=G_{0}$.

By iteration we obtain, for all $m \in \mathbb{N}$,

$$
G_{0} *\left(\left|B \nabla G_{0}\right|\right)^{* m}(x, y) \leq\left(2 C_{0}\|B\|\right)^{m} G_{0}(x, y) .
$$

Since $2 C_{0}\|B\|<1$, then the series

$$
\sum_{m=0}^{+\infty} G_{0} *\left(\left|B \nabla G_{0}\right|\right)^{* m}(x, y)
$$

is well defined and converges for $x \neq y \in D$. 
Let us define $G$ by

$$
G(x, y)= \begin{cases}\sum_{m=0}^{+\infty} G_{0} *\left(B \nabla G_{0}\right)^{* m}(x, y) & \text { if } x \neq y \\ +\infty & \text { if } x=y\end{cases}
$$

We will prove that $G$ is continuous. To this end we will show that, for any $m \geq 1, p_{m}(x, y) \equiv G_{0} *\left(\left|B \nabla G_{0}\right|\right)^{* m}(x, y)$ is continuous outside the diagonal.

Let $\left(x_{0}, y_{0}\right) \in D \times D$ with $x_{0} \neq y_{0}$. Put $\rho=\left|x_{0}-y_{0}\right|>0, E_{1}=$ $\bar{B}\left(x_{0}, \rho / 4\right)$ and $E_{2}=\bar{B}\left(y_{0}, \rho / 4\right)$. Since $B \in \mathcal{K}_{\mathrm{loc}}(D)$, then for any $\varepsilon>0$, there is $r \in(0, \rho / 4)$ such that, for $i=1,2$,

$$
\sup _{x \in E_{i}} \int_{D \cap(|x-z|<r)} \frac{|B(z)|}{|x-z|^{n-1}} d z<\varepsilon
$$

For $(x, y) \in B\left(x_{0}, r / 2\right) \times B\left(y_{0}, r / 2\right)$, we have

$$
\begin{aligned}
p_{1}(x, y) \equiv & \int_{D} G_{0}(x, z)|B(z)|\left|\nabla_{z} G_{0}\right|(z, y) d z \\
= & \int_{D \cap(|x-z|<r)} \cdots d z+\int_{D \cap(|y-z|<r)} \cdots d z \\
& +\int_{D \cap(|x-z| \geq r) \cap(|y-z| \geq r)} \cdots d z \\
\equiv & p_{11}(x, y)+p_{12}(x, y)+p_{13}(x, y) .
\end{aligned}
$$

By Theorem 2.1, we have

$$
\begin{aligned}
p_{11}(x, y) & \equiv \int_{D \cap(|x-z|<r)} G_{0}(x, z)|B(z)|\left|\nabla_{z} G_{0}\right|(z, y) d z \\
& \leq \frac{C}{\rho^{n-1}} \int_{D \cap(|x-z|<r)} G_{0}(x, z)|B(z)| d z \\
& \leq \frac{C}{\rho^{n-1}} \int_{D \cap(|x-z|<r)} \frac{|B(z)|}{|x-z|^{n-1}} d z \leq \frac{C}{\rho^{n-1}} \varepsilon .
\end{aligned}
$$


In the same way, we have

$$
\begin{aligned}
p_{12}(x, y) & \equiv \int_{D \cap(|y-z|<r)} G_{0}(x, z)|B(z)|\left|\nabla_{z} G_{0}\right|(z, y) d z \\
& \leq \frac{C}{\rho^{n-2}} \int_{D \cap(|y-z|<r)}|B(z)|\left|\nabla_{z} G_{0}\right|(z, y) d z \\
& \leq \frac{C}{\rho^{n-2}} \int_{D \cap(|y-z|<r)} \frac{|B(z)|}{|x-z|^{n-1}} d z \leq \frac{C}{\rho^{n-2}} \varepsilon .
\end{aligned}
$$

On the other hand, for $|x-z| \geq r$ and $|y-z| \geq r$, we have

$$
G_{0}(x, z)|B(z)|\left|\nabla_{z} G_{0}\right|(z, y) \leq \frac{C}{r^{n-1}}|B(z)| \Gamma(x, z)
$$

and

$$
\left|x_{0}-z\right| \leq|x-z|+\left|x-x_{0}\right| \leq \frac{3}{2}|x-z|
$$

which imply

$$
G_{0}(x, z)|B(z)|\left|\nabla_{z} G_{0}\right|(z, y) \leq \frac{C}{r^{n-1}}|B(z)| \Gamma\left(x_{0}, z\right) .
$$

Since

$$
\int_{D}|B(z)| \Gamma\left(x_{0}, z\right) d z \leq\|B\|<\infty
$$

then by the dominated convergence theorem we deduce that

$$
\lim _{(x, y) \rightarrow\left(x_{0}, y_{0}\right)} p_{13}(x, y)=p_{13}\left(x_{0}, y_{0}\right) .
$$

Combining (5.3)-(5.6), we then deduce that $p_{1}$ is continuous in $\left(x_{0}, y_{0}\right)$.

Clearly, by using the equality $p_{m}(x, y)=p_{m-1} *\left|B \nabla G_{0}\right|(x, y)$ and the same arguments as above, we prove the continuity of $p_{m}$ outside the diagonal. Therefore by (5.2), it follows that $G$ is continuous.

Moreover, since $G_{0}(\cdot, y)$ vanishes on $\partial D$, then by $(5.2), G(\cdot, y)$ satisfies the same property, and, for all $y \in D$, we have

$$
G(\cdot, y)=G_{0}(\cdot, y)+\int_{D} G(\cdot, z) B(z) \nabla_{z} G_{0}(z, y) d z \quad \text { on } \quad D \backslash\{y\}
$$

which implies

$$
L G(\cdot, y)=-\varepsilon_{y}
$$


in the distributional sense. Thus $G$ is a continuous Green function for $L$ on $D$. From (5.2) we also have, for all $x \neq y \in D$,

$$
\begin{aligned}
\left|G(x, y)-G_{0}(x, y)\right| & \leq \sum_{m=1}^{+\infty} G_{0} *\left(\left|B \nabla G_{0}\right|\right)^{* m}(x, y) \\
& \leq \sum_{m=1}^{+\infty}\left(2 C_{0}\|B\|\right)^{m} G_{0}(x, y) \\
& =\frac{2 C_{0}\|B\|}{1-2 C_{0}\|B\|} G_{0}(x, y) .
\end{aligned}
$$

By recalling that $G=G_{0}=+\infty$ on the diagonal and $4 C_{0}\|B\|<1$, we obtain

$$
\left(\frac{1-4 C_{0}\|B\|}{1-2 C_{0}\|B\|}\right) G_{0} \leq G \leq\left(\frac{1}{1-2 C_{0}\|B\|}\right) G_{0} .
$$

Conversely, assume that there exists a continuous Green function $\tilde{G}$ for $L$ on $D$ which is comparable to $G_{0}$. By definition, for all $y \in D, L \tilde{G}(\cdot, y)=$ $-\varepsilon_{y}$ and $\tilde{G}(\cdot, y)=0$ on $\partial D$. This implies

$$
\tilde{G}(\cdot, y)=G_{0}(\cdot, y)+\int_{D} \tilde{G}(\cdot, z) B(z) \nabla_{z} G_{0}(z, y) d z \quad \text { a.e. on } D \text {. }
$$

Since $\tilde{G}$ and $G_{0}$ are continuous, it follows that

$$
\tilde{G}(\cdot, y)=G_{0}(\cdot, y)+\int_{D} \tilde{G}(\cdot, z) B(z) \nabla_{z} G_{0}(z, y) d z \quad \text { on } \quad D \backslash\{y\} .
$$

By iteration we obtain, for all $p \in \mathbb{N}$

$\tilde{G}(\cdot, y)=\sum_{m=0}^{p} G_{0} *\left(B \nabla G_{0}\right)^{* m}(\cdot, y)+\tilde{G} *\left(B \nabla G_{0}\right)^{*(p+1)}(\cdot, y) \quad$ on $\quad D \backslash\{y\}$.

Since $\tilde{G}$ is comparable to $G_{0}$, then by $(5.2)$, we have

$$
\tilde{G} *\left(\left|B \nabla G_{0}\right|\right)^{*(p+1)}(\cdot, y) \leq\left(2 C_{0}\|B\|\right)^{p+1} G_{0}(\cdot, y) .
$$

By recalling that $2 C_{0}\|B\|<1$ and letting $p \rightarrow \infty$, we obtain

$$
\tilde{G}(\cdot, y)=\sum_{m=0}^{+\infty} G_{0} *\left(B \nabla G_{0}\right)^{* m}(\cdot, y) \quad \text { on } \quad D \backslash\{y\} .
$$

By the comparability of $\tilde{G}$ to $G_{0}$, we also have $\tilde{G}=+\infty$ on the diagonal. Thus $\tilde{G}=G$ which completes the proof. 
Remark 5.2. By the Hölder inequality, it is easy to see that the class $\mathcal{K}_{\text {loc }}(D)$ contains all the spaces $L^{p}(D)$ with $p>n$ and so in contrast to Theorem 9.1' in Ancona's paper [5], our result (Theorem 5.1) provide a constant $C$ which does not depend on the parameter $p$.

\section{Estimates of the $L$-harmonic measure}

In [10], Dahlberg proved that the Laplace harmonic measure and the surface measure are equivalent on the boundary of a Lipschitz domain. This result was later extended by Ancona [4] to a class of uniformly elliptic operators with bounded Hölder continuous coefficients. Subsequently, Cranston and Zhao [9] studied the problem for the operator $\frac{1}{2} \Delta+B(x) \cdot \nabla_{x}$ with $B$ and $|B|^{2}$ are in the Kato classes $K_{n+1}$ and $K_{n}$, respectively. In [5], Ancona also deduced the equivalence of the harmonic measure and the surface measure (see Theorems 9.3 and 9.4). In this section, we will investigate the same problem for the operator $L=\operatorname{div}\left(A(x) \nabla_{x}\right)+B(x) \cdot \nabla_{x}$ with the drift term $B$ in $\mathcal{K}_{\text {loc }}(D)$. We have shown in Section 5 that when $B \in \mathcal{K}_{\text {loc }}(D)$ with $\|B\| \leq 1 / 4 C_{0}$, the operator $L$ admits a continuous Green function and so it has positive continuous solutions. By the standard theory $[\mathbf{3}],[\mathbf{8}]$, we have the existence and uniqueness of the $L$-Martin Poisson kernel and the unique solvability of the Dirichlet problem with continuous boundary data. In what follows, we estimate the $L$-Poisson kernel and the $L$-harmonic measure.

Theorem 6.1. Let $B \in \mathcal{K}_{\text {loc }}(D)$ with $\|B\| \leq 1 / 4 C_{0}$. Then, the L-Poisson kernel $P$ on $D$ satisfies the following estimates: there exists a constant $C=C(n, \mu, D,\|B\|)>0$, such that

$$
\frac{1}{C} \frac{d(x)}{|x-Q|^{n}} \leq P(x, Q) \leq C \frac{d(x)}{|x-Q|^{n}},
$$

for all $x \in D$ and $Q \in \partial D$.

Proof: Since $D$ is of $C^{1,1}$-boundary, then by the divergence theorem, we have

$$
P(x, Q)=\frac{\partial G(x, Q)}{\partial N_{Q}},
$$

where $N_{Q}=A(Q) n_{Q}$ with $n_{Q}$ is the unit inner normal to $\partial D$ at $Q$.

We write $N_{Q}=T_{Q}+a(Q) n_{Q}$, where $T_{Q}$ is a tangential vector field to $\partial D$ at $Q$.

Therefore

$$
\frac{\partial G(x, Q)}{\partial N_{Q}}=\frac{\partial G(x, Q)}{\partial T_{Q}}+a(Q) \frac{\partial G(x, Q)}{\partial n_{Q}} .
$$


Since $G(x, \cdot) \equiv 0$ on $\partial D$, then

and so

$$
\frac{\partial G(x, Q)}{\partial T_{Q}} \equiv \frac{\partial}{\partial T_{Q}}(G(x, \cdot))(Q)=0
$$

$$
\begin{aligned}
\frac{\partial G(x, Q)}{\partial N_{Q}} & =a(Q) \frac{\partial G(x, Q)}{\partial n_{Q}} \\
& \equiv a(Q) \lim _{r \rightarrow 0^{+}} \frac{G\left(x, Q+r n_{Q}\right)}{r}
\end{aligned}
$$

On the other hand, by recalling that, for $r>0$ small, $d\left(Q+r n_{Q}\right)=$ $r$, it follows from the comparability of $G$ to $G_{0}$ and the estimates in Theorem 2.1, that

$$
\frac{1}{C} \frac{d(x)}{\left|x-Q-r n_{Q}\right|^{n}} \leq \frac{G\left(x, Q+r n_{Q}\right)}{r} \leq C \frac{d(x)}{\left|x-Q-r n_{Q}\right|^{n}} .
$$

By noting that $1 / \mu \leq\left\langle A(Q) n_{Q}, n_{Q}\right\rangle \leq \mu$, combining (6.1), (6.2) and letting $r$ to 0 we obtain the estimates stated in the theorem.

For $x \in D$, let $m_{x}$ denotes the $L$-harmonic measure at $x$ on $\partial D$ and $\sigma$ the surface measure on $\partial D$. We have the following:

Corollary 6.2. Let $B \in \mathcal{K}_{\text {loc }}(D)$ with $\|B\| \leq 1 / 4 C_{0}$. Then, the measures $m_{x}$ and $\sigma$, are equivalent on $\partial D$ with densities in $L^{\infty}(\partial D)$.

Proof: For any Borel subset $E \subset \partial D$, we have

$$
m_{x}(E)=\int_{E} P(x, Q) d \sigma(Q),
$$

and so by Theorem 6.1 , it follows that

$$
\frac{1}{C} \frac{d(x)}{d(D)^{n}} \sigma(E) \leq m_{x}(E) \leq \frac{C}{d(x)^{n-1}} \sigma(E) .
$$

Remarks.

1. Since the matrix $A=A(x) \in C^{0,1}(D)$, we can also write $L$ in the non-divergence form

$$
L=\sum_{i, j=1}^{n} a_{i j}(x) \frac{\partial^{2}}{\partial x_{i} \partial x_{j}}+\left(B+B^{\prime}\right)(x) \cdot \nabla_{x},
$$

where $B^{\prime}=\left(B_{1}^{\prime}, \ldots, B_{n}^{\prime}\right)$ with $B_{j}^{\prime}=\sum_{i=1}^{n} \frac{\partial a_{i j}}{\partial x_{i}}(x) \in L^{\infty}(D)$.

From this observation we see that our results are valid for the operators in the non-divergence form as well. 
2. Although the adjoint operator $L^{*}=\operatorname{div}\left(A(x) \nabla_{x}\right)-\operatorname{div}(B(x) \cdot)$ has a different structure from $L$, by the equality $G^{*}(x, y)=G(y, x)$, where $G^{*}$ is the $L^{*}$-Green function on $D$, we deduce that our results are also true for $L^{*}$.

3. By Theorem 3.3 in $[\mathbf{1 1}]$ and the arguments used to prove the lower bound (Theorem 1 in [27]), the estimates stated in Theorem 2.1 are also valid on domains satisfying the uniformly exterior ball condition. So, our results are also true for this class of domains which contains the class of $C^{1,1}$-domains (see [7, p. 179]).

4. As is shown in [8] and [23], the comparability of the Green functions yields some potential theoretic results for $L$ which are known to hold for the Laplace operator $\Delta$. For instance we deduce interior and boundary Harnack principles for nonnegative $L$-solutions, Hölder continuity of bounded solutions, integral representations of nonnegative $L$-solutions, etc, . . .

Acknowledgements. We would like to sincerely thank the referee for her/his valuable comments and for drawing our attention to the related results in the paper [5].

\section{References}

[1] H. Aikawa, Norm estimate of Green operator, perturbation of Green function and integrability of superharmonic functions, Math. Ann. 312(2) (1998), 289-318.

[2] M. Aizenman And B. Simon, Brownian motion and Harnack inequality for Schrödinger operators, Comm. Pure Appl. Math. 35(2) (1982), 209-273.

[3] A. Ancona, Principe de Harnack à la frontiére et théorème de Fatou pour un opérateur elliptique dans un domaine lipschitzien, Ann. Inst. Fourier (Grenoble) 28(4) (1978), 169-213.

[4] A. Ancona, Comparaison des mesures harmoniques et des fonctions de Green pour des opérateurs elliptiques sur un domaine lipschitzien, C. R. Acad. Sci. Paris Sér. I Math. 294(15) (1982), $505-508$.

[5] A. Ancona, First eigenvalues and comparison of Green's functions for elliptic operators on manifolds or domains, J. Anal. Math. 72 (1997), 45-92.

[6] A. Boukricha, W. Hansen and H. Hueber, Continuous solutions of the generalized Schrödinger equation and perturbation of harmonic spaces, Exposition. Math. 5(2) (1987), 97-135. 
[7] K. L. Chung And Z. X. ZhaO, "From Brownian motion to Schrödinger's equation", Grundlehren der Mathematischen Wissenschaften 312, Springer-Verlag, Berlin, 1995.

[8] M. Cranston, E. Fabes and Z. X. Zhao, Conditional gauge and potential theory for the Schrödinger operator, Trans. Amer. Math. Soc. 307(1) (1988), 171-194.

[9] M. Cranston and Z. X. Zhao, Conditional transformation of drift formula and potential theory for $\frac{1}{2} \Delta+b(\cdot) \cdot \nabla$, Comm. Math. Phys. 112(4) (1987), 613-625.

[10] B. E. J. DAhlberg, Estimates of harmonic measure, Arch. Rational Mech. Anal. 65(3) (1977), 275-288.

[11] M. Grüter And K.-O. Widman, The Green function for uniformly elliptic equations, Manuscripta Math. 37(3) (1982), 303-342.

[12] W. HANSEn, Normalized solutions of Schrödinger equations with potentially bounded measures, Potential Anal. 21(2) (2004), 99-135.

[13] W. Hansen, Global comparison of perturbed Green functions, Preprint 03-046, Bibos Bielefeld (2003).

[14] H. Hueber, A uniform estimate for Green functions on $C^{1,1}$-domains, Bibos Publication Universität Bielefeld (1986).

[15] H. Hueber And M. Sieveking, Uniform bounds for quotients of Green functions on $C^{1,1}$-domains, Ann. Inst. Fourier (Grenoble) 32(1) (1982), 105-117.

[16] N. J. Kalton And I. E. Verbitsky, Nonlinear equations and weighted norm inequalities, Trans. Amer. Math. Soc. 351(9) (1999), 3441-3497.

[17] M. Murata, Structure of positive solutions to $(-\Delta+V) u=0$ in $\mathbb{R}^{n}$, Duke Math. J. 53(4) (1986), 869-943.

[18] M. Murata, Semismall perturbations in the Martin theory for elliptic equations, Israel J. Math. 102 (1997), 29-60.

[19] Y. Pinchover, On the equivalence of Green functions of second order elliptic equations in $\mathbb{R}^{n}$, Differential Integral Equations 5(3) (1992), 481-493.

[20] Y. Pinchover, Maximum and anti-maximum principles and eigenfunctions estimates via perturbation theory of positive solutions of elliptic equations, Math. Ann. 314(3) (1999), 555-590.

[21] Y. Pinchover, Criticality and ground states for second-order elliptic equations, J. Differential Equations 80(2) (1989), 237-250. 
[22] L. Riahi, Comparison of Green functions for generalized Schrödinger operators on $C^{1,1}$-domains, JIPAM. J. Inequal. Pure Appl. Math. 4(1) (2003), no. 1, Article 22, 14 pp. (electronic).

[23] L. Riahi, The $3 G$-inequality for general Schrödinger operators on Lipschitz domains, Manuscripta Math. 116(2) (2005), 211-227.

[24] M. Selmi, Comparaison des noyaux de Green sur les domaines $C^{1,1}$, Rev. Roumaine Math. Pures Appl. 36(1-2) (1991), 91-100.

[25] Yu A. Semenov, Stability of $L^{p}$-spectrum of generalized Schrödinger operators and equivalence of Green's functions, Internat. Math. Res. Notices 12 (1997), 573-593.

[26] K.-O. Widman, Inequalities for the Green function and boundary continuity of the gradient of solutions of elliptic differential equations, Math. Scand. 21 (1967), 17-37.

[27] Z. X. Zнно, Green function for Schrödinger operator and conditioned Feynman-Kac gauge, J. Math. Anal. Appl. 116(2) (1986), 309-334.

[28] Z. X. Zhaо, Subcriticality and gaugeability of the Schrödinger operator, Trans. Amer. Math. Soc. 334(1) (1992), 75-96.

Department of Mathematics

Faculty of Sciences of Tunis

Campus Universitaire 1060

Tunis

Tunisia

E-mail address: Ifra.Abdoul@fst.rnu.tn

E-mail address: Lofti.Riahi@fst.rnu.tn

Primera versió rebuda el 26 de febrer de 2004, darrera versió rebuda el 30 de juliol de 2004. 